\title{
ORIGINAL
}

\section{Gallbladder neuroendocrine neoplasm: a case report and critical evaluation of WHO classification}

\author{
Giulia Meoni ${ }^{1)}$, Lorenzo Antonuzzo ${ }^{1)}$, Luca Messerini ${ }^{2)}$, Elisa Giommoni ${ }^{1)}$, Andrea Muto ${ }^{1)}$, Paolo Petreni ${ }^{1)}$, \\ Agnese Vannini ${ }^{1)}$, Alice Lunghi ${ }^{1)}$, Elena Molinara ${ }^{1)}$ and Francesco Di Costanzo ${ }^{1)}$ \\ 1) Medical Oncology Unit, Azienda Ospedaliero-Universitaria Careggi, Florence 50134, Italy \\ 2) Histopathology and Molecular Pathology, Section of Surgery, Experimental and Clinical Medicine, University of Florence, \\ Florence 50134, Italy
}

\begin{abstract}
Gallbladder neuroendocrine neoplasms (GB-NENs) are rare. The majority of GB-NENs are poorly differentiated, with increased mitotic activity and clinically aggressive course. Surgery is the only curative approach and the optimal medical treatment is uncertain. In this report we describe the case of a woman affected by metastatic well differentiated GB-NEN with increased Ki 67. The patient underwent surgical removal of the gallbladder neoplasm and showed disease recurrence with pulmonary and liver metastases. After achieving a partial chemotherapy response, the patient rapidly died due to progressive disease. This case raises important issues. Well differentiated NENs with a high proliferative index are not included as a specific entity in any of the most widely used nomenclature systems. Moreover considering the proliferative index of the disease, it is reasonable to consider the patient a candidate for chemotherapy. Nevertheless, recently published papers raise the possibility that well differentiated NENs and specific proliferative index cutoff can predict low chemosensitivity in patients with highly proliferative neuroendocrine carcinoma.
\end{abstract}

Key words: Neuroendocrine gallbladder carcinoma, Neuroendocrine neoplasms, WHO classification, Grade of differentiation, Proliferative index

PRIMARY gallbladder neuroendocrine neoplasms (GB-NENs) are an extremely rare disease, representing $0.5 \%$ of all NENs [1]. In the Surveillance, Epidemiology and End Results (SEER) registry only 278 cases of GB-NENs have been reported from 1973 to 2005 [2]. They are more frequent among females $(68 \%)$ and the age at presentation ranges from 26 to 79 years [3]. It seems certain that neuroendocrine (NE) epithelial cells of gallbladder origin were from mucosal areas which underwent intestinal and/or gastric metaplasia due to chronic inflammation from cholelithiasis [4]. The vast majority of GB-NENs are poorly differentiated tumors, with increased mitotic activity and clinically aggressive course (5 year overall survival of $20 \%$ )

Submitted Apr. 25, 2014; Accepted Jul. 3, 2014 as EJ14-0191 Released online in J-STAGE as advance publication Aug. 2, 2014 Correspondence to: Giulia Meoni M.D., Azienda OspedalieroUniversitaria Careggi, Medical Oncology Unit, Largo Brambilla 3, Florence 50134, Italy. E-mail: giulia.meoni@gmail.com Abbreviations: GB-NENs, gallbladder neuroendocrine neoplasms; SEER, Surveillance Epidemiology and End Results; NE, neuroendocrine; WHO, World Health Organization; NEC, neuroendocrine
[2]. Whereas surgery is the only curative approach, the optimal medical treatment of this disease is still uncertain. The World Health Organization (WHO) 2010 classification identifies three grade of NENs: low grade neoplasms (G1, Ki-67 index $<3 \%$ ) with differentiated pathologic features, high grade neoplasms G3, (Ki-67 index $>20 \%$ ) with poorly differentiated pathologic features, intermediate grade neoplasms (G2, Ki-67 index $\geq 3 \%$ and $\leq 20 \%$ ) with moderately differentiated pathologic features. In this report we describe the case of a woman affected by metastatic well-differentiated GB-NEN with increased Ki-67 (40\%). The patient developed rapidly pulmonary and liver metastatic disease. After an initial good chemotherapy response, the

carcinoma; CgA, chromogranin A; NSE, neuronspecific enolase; ECOG PS, Eastern Cooperative Oncology Group Performance Status; DFS, disease free survival; PR, partial response; ENETS, European Neuroendocrine Tumor Society; GEP-NETs, gastro entero pancreatic neuroendocrine tumors; WDNET, well-differentiated neuroendocrine tumor; LCNEC, large-cell neuroendocrine carcinoma; GI-NEC, gastrointestinal neuroendocrine carcinoma; PFS, progression free survival 


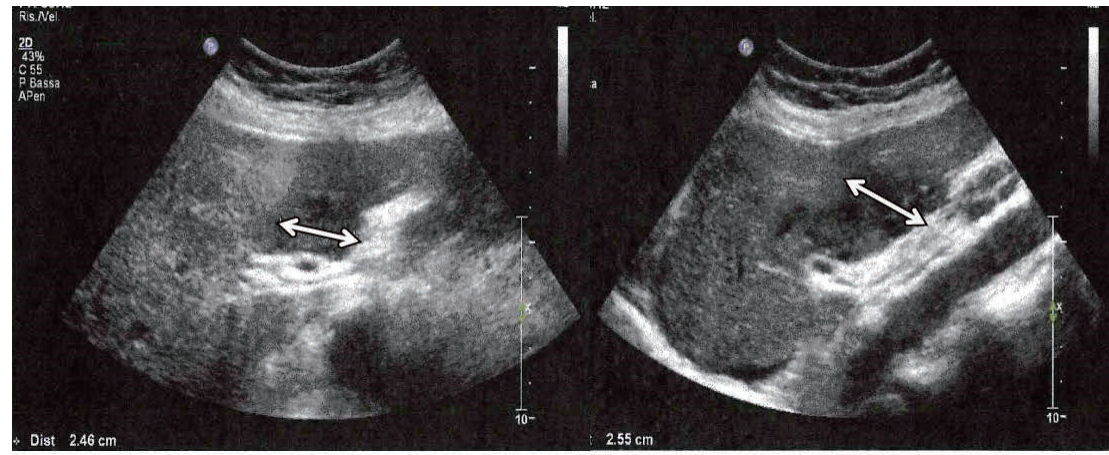

Fig. 1 Preoperative liver ultrasound

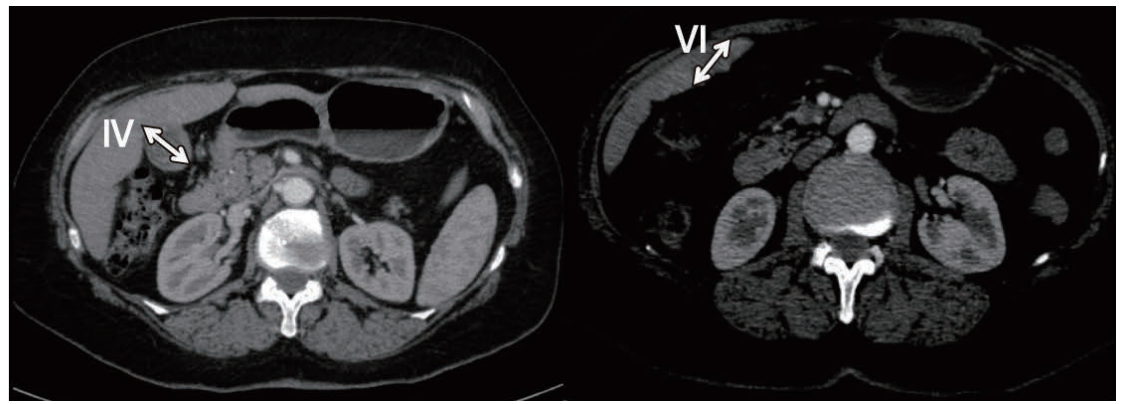

Fig. 2 Preoperative abdominal CT scan: CT scan of the abdomen confirmed two hepatic lesions: the first measuring $23 \mathrm{~mm}$ in segment IV and the second measuring $26 \mathrm{~mm}$ in segment VI.

patient died due to liver failure. This case raises interesting topics of discussion among NENs. First of all, histological features make this GB-NEN unclassifiable with the most widely used systems. Moreover, the discordant histopathological aspects raise important therapeutic issues which are partially addressed by recent published papers, but still remain unresolved.

\section{Case Presentation}

The case is about a 66-year-old Caucasian woman with complaints of recurrent abdominal pain for several months. As prescribed by her general practitioner, the patient underwent abdomen ultrasound, which revealed the presence of two solid hepatic nodules (Fig. 1). CT scan of the abdomen confirmed two hepatic lesions: the first measuring $23 \mathrm{~mm}$ in segment IV and the second measuring $26 \mathrm{~mm}$ in segment VI (Fig. 2). The patient was subsequently referred to a surgeon and in February 2010 she underwent cholecystectomy, left hepatic lobe resection and D2 lymphadenectomy. The pathologist found at gross examination an irregular nodular mass, measuring $3.5 \times 3 \mathrm{~cm}$, in the context of the gallbladder wall. The lesion was entirely

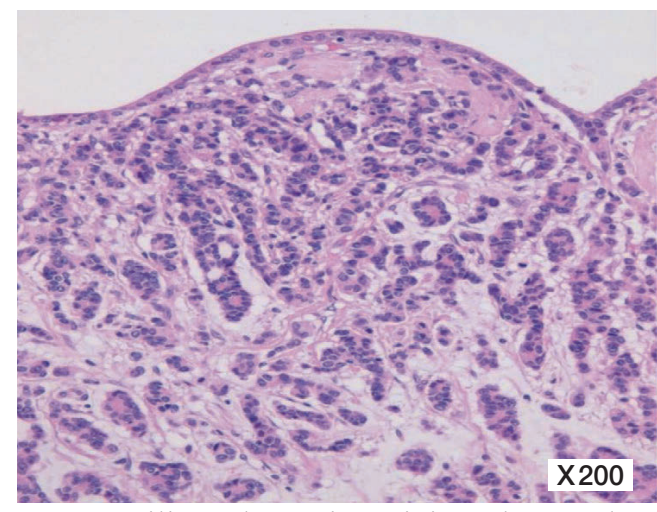

Fig. 3 Hematoxilin and Heosin staining: the neoplastic cell diffusely involved the gallbladder, but the surface epithelium was intact.

sampled. Histologically the tumor was composed of glandular and acinar structures which diffusely infiltrated the gallbladder and were diffused to the liver and to the loco-regional lymph-nodes, but the surface epithelium of the gallbladder was intact (Fig. 3). Immunohistochemical studies revealed a strong positivity for both chromogranin A (CgA) (Fig. 4) and synaptophisyn, and any exocrine tumoral component was excluded. The proliferative activity of tumor cells was 


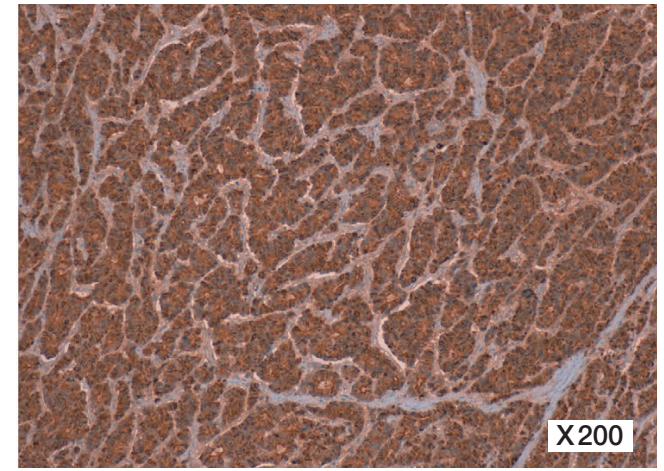

Fig. 4 Chromogranin A staining: diffuse homogeneous pattern of the immunoreaction in the neoplastic cells.

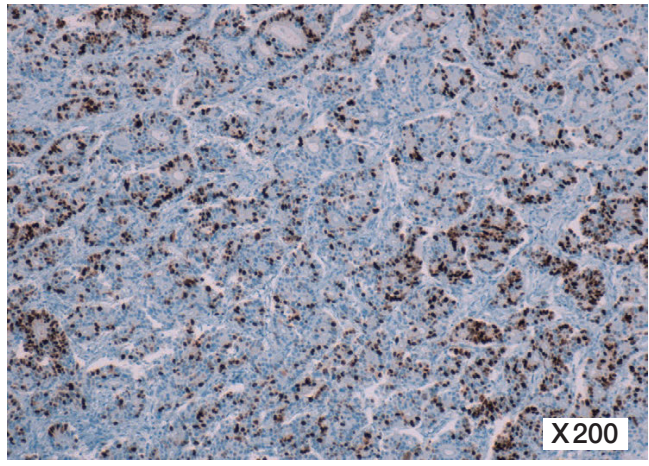

Fig. 5 Ki-67 proliferative index: high proliferative rate (40\%) with an unhomogeneous distribution of the immunostainig in the neoplastic cells.

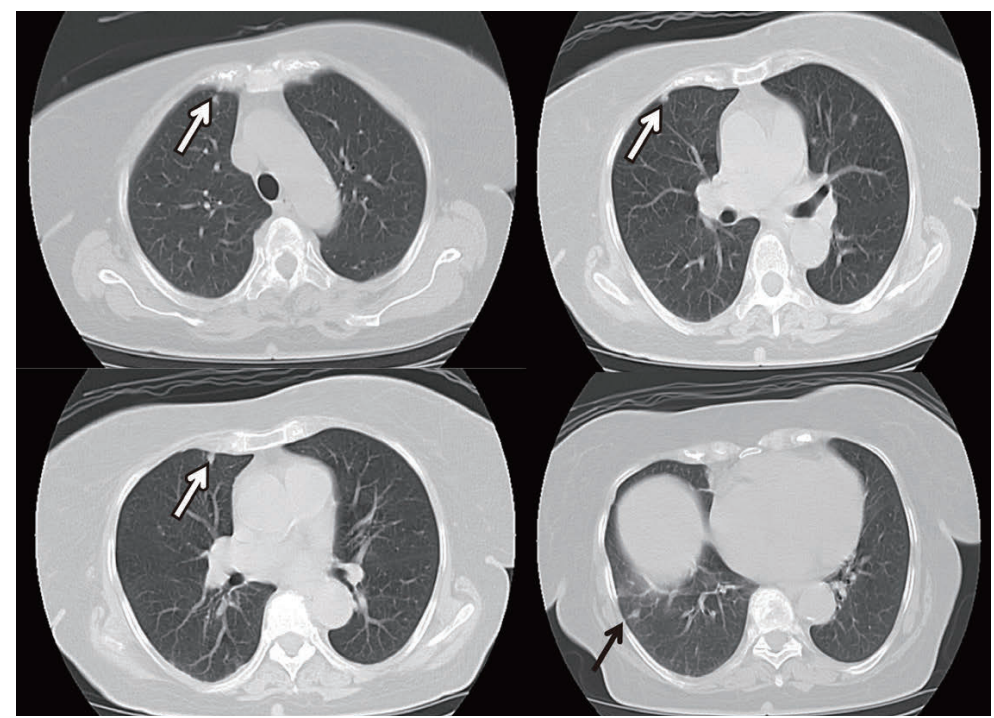

Fig. 6 Baseline CT scan, pulmonary metastases

evaluated by Ki-67 was $40 \%$ (Fig. 5), therefore we considered the tumor as a well-differentiated neuroendocrine carcinoma (NEC) G3 according to the WHO 2010 criteria. Post-surgical staging with CT scan of the thorax and abdomen and ${ }^{111}$ In-pentetreotide scintigraphy $\left(\right.$ Octreoscan ${ }^{\circledR}$ ) was negative for residual disease. Blood levels of CgA and neuronspecific enolase (NSE) were within normal limits. Given the lack of evidence as to the efficacy of any adjuvant medical treatment, in October 2010 patient started follow-up. Recurrence of disease was diagnosed in February 2011, as CT scan revealed pulmonary bilateral small metastases (Fig. 6) and multiple liver metastases (Fig. 7). A new Octreoscan ${ }^{\circledR}$ was negative for somatostatin receptor expression. CgA measured $282 \mathrm{ng} / \mathrm{mL}$ (normal range $10.0-185.0 \mathrm{ng} / \mathrm{mL}$ ). The patient was in good gen- eral condition, Eastern Cooperative Oncology Group Performance Status (ECOG PS) 0 and with no major comorbidities. Given the short disease free survival (DFS) interval, the burden of disease and the high proliferative activity of the tumor (Ki-67 40\%), in February 2011 the patient started first line chemotherapy with Cisplatin $75 \mathrm{mg} / \mathrm{mq}$ IV for 1 day and Etoposide 100 $\mathrm{mg} / \mathrm{mq}$ IV for 1-3 days once every three weeks. A total of 6 cycles were administered until August 2011. The patient experienced afebrile neutropenia G4 (Common Terminology Criteria for Adverse Events version 4.0) after the first cycle, which required $25 \%$ dose reduction of the next chemotherapy administrations. Nausea $\mathrm{G} 2$ and vomit G1 were also present as side effects. The CT scan performed after the third cycle showed partial response (PR), which was confirmed after three more 


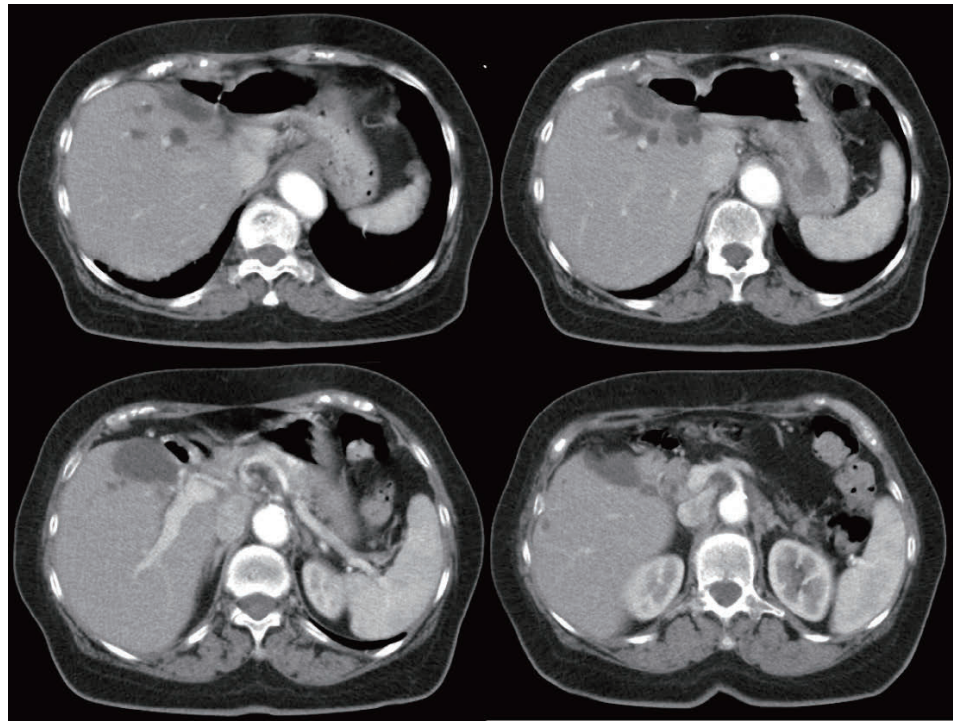

Fig. 7 Baseline CT scan, liver metastases

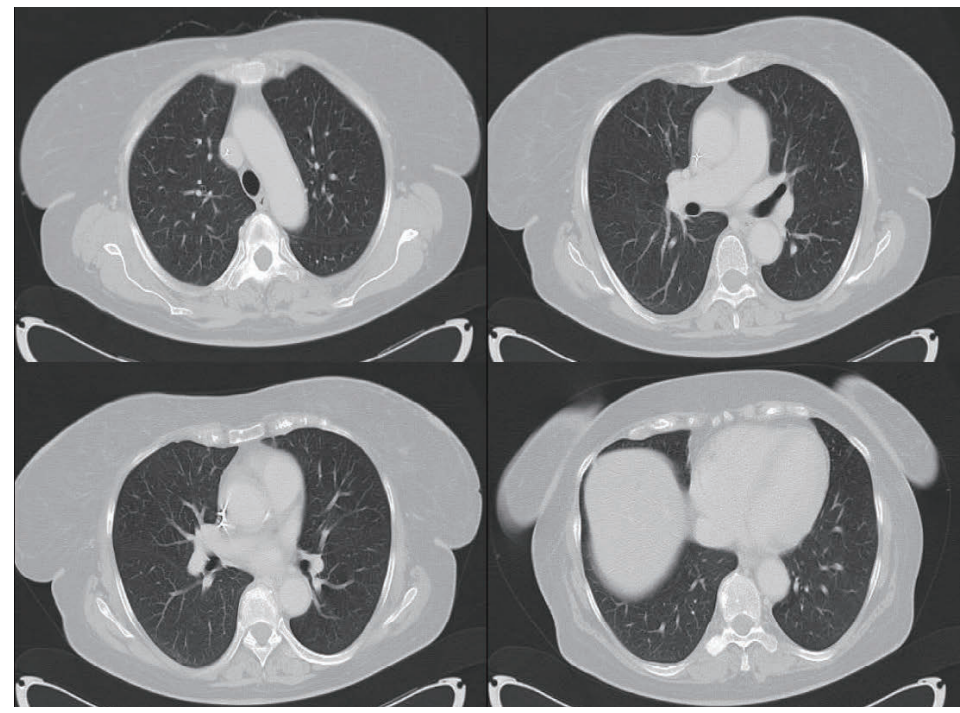

Fig. 8 CT scan after chemotherapy cycle VI, pulmonary metastases

cycles of chemotherapy (Figs. 8, 9). CgA decreased to $100 \mathrm{ng} / \mathrm{ml}$ after the last cycle. In December 2011 the patient developed rapid PS deterioration and jaundice. Liver ultrasound revealed complete hepatic subversion, due to the presence of large scattered liver metastasis. The patient died in January 2012 due to liver failure.

\section{Discussion}

In this report we describe the case of a woman affected by metastatic well-differentiated GB-NEN with increased Ki-67. To our knowledge this is the first case reported in literature with such histopathological features. Nevertheless the mitotic index and the differentiation have not always been reported together in the description of GB-NENs cases.

The majority of GB-NENs are diagnosed during histological examination at autopsy or after surgery for cholelithiasis or other suspected biliary pathology [5-7]. Considering the pathogenesis of GB-NENs, cholelithiasis and chronic gallbladder wall inflammation are associated with GB-NENs in virtually all published 


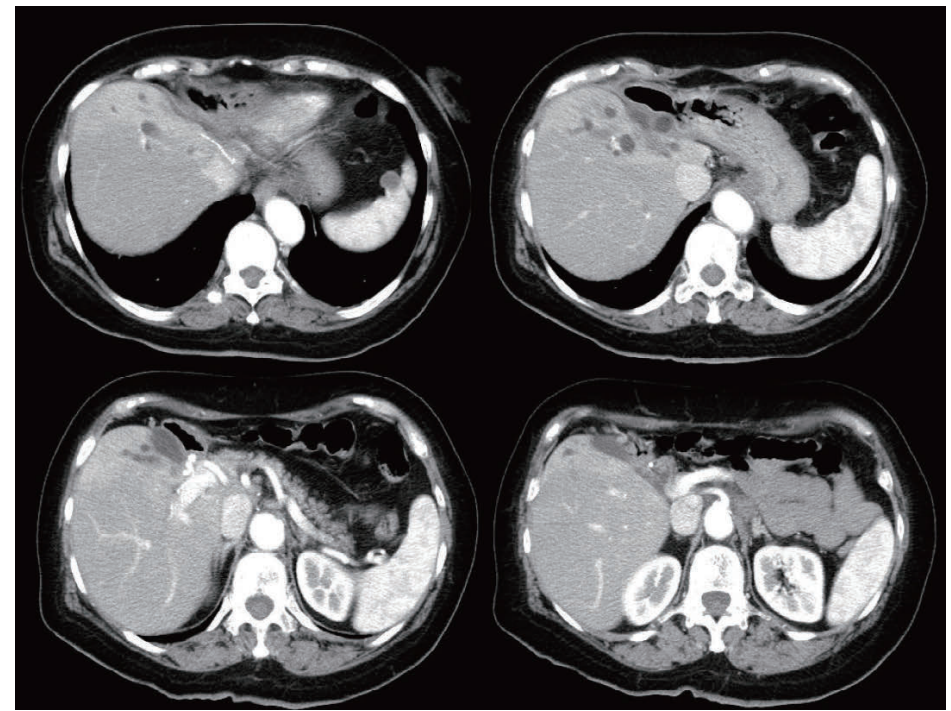

Fig. 9 CT scan after chemotherapy cycle VI, liver metastases

reports. Moreover it could be plausible that NE cells, usually absent in the gallbladder epithelium, derive from local multipotent gastrointestinal stem cells [8], from ectopic pancreatic tissue [9] or from nerve fibers located in the gallbladder wall [10].

Rarely GB-NENs are associated with carcinoid syndrome $(<1 \%)$.

The vast majority of GB-NENs are poorly differentiated tumors, with increased mitotic activity. In the Pan SEER 9 (1973-2005) database, the differentiation was reported in 41 cases and it was observed that $26.3 \%$ were poorly differentiated and $63.4 \%$ were undifferentiated/anaplastic tumors [2].

The European Neuroendocrine Tumor Society (ENETS) and the WHO 2010 grading system for gastro entero pancreatic neuroendocrine tumors (GEPNETs) are validated only for stomach, duodenum and pancreas NENs [11], but they are widely used even to classify GEP-NEN of different origins. These classifications systems identify three grades of NENs: low grade neoplasms (G1, Ki-67 index $<3 \%$ ) with an indolent behavior, high grade neoplasms (G3, Ki-67 index $>20 \%$ ) with an aggressive behavior and intermediate grade neoplasms (G2, Ki-67 index $\geq 3 \%$ and $\leq 20 \%$ ) with a moderately aggressive behavior [11]. Moreover, the ENETS and the WHO 2010 nomenclature systems identify two different families of NENs, the first including well and moderately differentiated neuroendocrine tumors (G1 and G2 NETs) and the second including poorly differentiated G3 NEC [11].
Although not encompassed in these two big groups of NENs, the existence of well-differentiated high grade NENs, such as the GB-NEN reported in this manuscript, is well documented.

Scoazec and colleagues prospectively evaluated the agreement between grading and differentiation among 1340 NENs of different origin according to the WHO 2010 system [12]. The authors found that, among 104 G3 NEC, 21 (20\%) were well-differentiated G3 NEC.

Recently Vélayoudom-Céphise and colleagues reviewed 166 cases of G3 non-small-cell-type NET [13]. Tumors were classified as well-differentiated NET (G3-WDNET) in $42.8 \%$ of cases and poorly differentiated, large-cell NEC (G3-LCNEC) in 57.2\% of cases. Complete or partial response to cisplatin was observed in $31 \%$ of cases, all classified as G3-LCNEC. The median OS was 41 months for G3-WDNET but only 17 months for G3-LCNEC. The patient of our case, which corresponds to the G3-WDNET population, showed short OS and a good chemotherapy response, not confirming therefore the observation of Vélayoudom-Céphise et al.

The recently published NORDIC NEC study retrospectively analyzed 305 patients with metastatic gastrointestinal neuroendocrine carcinoma (GI-NEC) or unknown primary tumor with predominantly GI metastases [14].

Based on this study, Ki- $67<55 \%$ could be a positive prognostic factor and a negative predictive factor to platinum based chemotherapy. The patient of our report, had 40\% Ki-67 proliferative index and achieved 
a very good response to platinum base chemotherapy but a very short survival of 10 months. Thus these data are not in accordance with the retrospective observation of the NORDIC study.

The proliferative index and the differentiation of the tumor represent both strong and validated prognostic factors $[1,15,16]$. High grade NEC have a poor prognosis as do poorly differentiated tumors. Little evidence exists about the prognosis of those NENs with discordant grade and differentiation. In this case report the proliferative index, instead of the tumor differentiation, influenced mainly the biological behavior of the disease and the prognosis of the patient.

\section{Conclusion}

In conclusion, our case is about a patient affected by a well-differentiated highly proliferative metastatic GB-NEN which is a rare disease with peculiar histopathological features, that make this pathologic entity unclassifiable with the current nomenclature systems. Little evidence exists about the optimal medical treatment of this disease in the metastatic setting and patients show a dismal prognosis in virtually all cases reported.

\section{Disclosure}

None of the authors have any potential conflicts of interest associated with this research.

\section{References}

1. Yao JC, Hassan M, Phan A, Dagohoy C, Leary C, et al. (2008) One hundred years after "carcinoid": epidemiology of and prognostic factors for neuroendocrine tumors in 35,825 cases in the United States. J Clin Oncol 26: 3063-3072.

2. Eltawil KM, Gustafsson BI, Kidd M, Modlin IM (2010) Neuroendocrine tumors of the gallbladder: an evaluation and reassessment of management strategy. $J$ Clin Gastroenterol 44: 687-695.

3. Deehan DJ, Heys SD, Kernohan N, Eremin O (1993) Carcinoid tumour of the gall bladder: two case reports and a review of published works. Gut 34: 1274-1276.

4. Christie AC (1954) Three cases illustrating the presence of argentaffin (kultschitzky) cells in the human gallbladder. J Clin Pathol 7: 318-321.

5. Nishigami T, Yamada M, Nakasho K, Yamamura M, Satomi M, et al. (1996) Carcinoid tumor of the gall bladder. Intern Med 35: 953-956.

6. Anjaneyulu V, Shankar-Swamalatha G, Rao SC (2007) Carcinoid tumor of the gall bladder. Ann Diagn Pathol 11: 113-116.

7. Ozawa K, Kinoshita M, Kagata Y, Matsubara O (2003) A case of double carcinoid tumors of the gallbladder. Dig Dis Sci 48: 1760-1761.

8. Pearse AG (1969) The cytochemistry and ultrastructure of polypeptide hormone-producing cells of the APUD series and the embryologic, physiologic and pathologic implications of the concept. J Histochem Cytochem 17: 303-313.

9. Marrano NN, Blevins LS, Gal AA, McGuire WP (1999) Pancreatic polypeptide hypersecretion associated with a neuroendocrine carcinoma of the gallbladder. Am J Med Sci 317: 55-58.
10. Uemura S, Pompolo S, Furness JB, Hardy KJ (1997) Nitric oxide synthase in neurons of the human gallbladder and its colocalization with neuropeptides. $J$ Gastroenterol Hepatol 12: 257-265.

11. Rindi G, Arnold R, Bosman FT (2010) Nomenclature and classification of neuroendocrine neoplasms of the digestive system. In: Bosman FT, et al. (ed.) WHO classification of tumours of the digestive system $\left(4^{\text {th }}\right)$. IARC Press, Lyon: 13-14.

12. Scoazec JY, Couvelard A, Monges G, Leteurtre E, Belleannee G, et al. (2012) Well-differentiated grade 3 digestive neuroendocrine tumors: Myth or reality - The PRONET study group. 2012 ASCO Annual Meeting, A4129 (Abstract).

13. Vélayoudom-Céphise FL, Duvillard P, Foucan L, Hadoux J, Chougnet CN, et al. (2013) Are G3 ENETS neuroendocrine neoplasms heterogeneous? Endocr Relat Cancer 20: 649-657.

14. Sorbye H, Welin S, Langer SW, Vestermark LW, Holt N, et al. (2013) Predictive and prognostic factors for treatment and survival in 305 patients with advanced gastrointestinal neuroendocrine carcinoma (WHO G3): the NORDIC NEC study. Ann Oncol 24: 152-160.

15. Jann H, Roll S, Couvelard A, Hentic O, Pavel M, et al. (2011) Neuroendocrine tumors of midgut and hindgut origin: tumor-node-metastasis classification determines clinical outcome. Cancer 117: 3332-3341.

16. Ferrone CR, Tang LH, Tomlinson J, Gonen M, Hochwald SN, et al. (2007) Determining prognosis in patients with pancreatic endocrine neoplasms: can the WHO classification system be simplified? J Clin Oncol 25: 5609-5615. 